\title{
Mutation-negative FAP patients with mRNA defects of APC
}

\author{
Michelle W Condie ${ }^{1}$, Therese MF Tuohy ${ }^{1 *}$, Patricia Shires ${ }^{2}$, Randall W Burt ${ }^{1,3}$, Deborah W Neklason ${ }^{1,4}$ \\ From 13th Annual Meeting of the Collaborative Group of the Americas on Inherited Colorectal Cancer \\ Honolulu, Hawaii, USA. 16-17 October 2009
}

\section{Background}

Familial adenomatous polyposis (FAP) is a colon cancer syndrome with a prevalence of 1:10:000, hallmarked by 100 s to 1000 s of precancerous colonic polyps and nearly $100 \%$ lifetime risk of developing colon cancer at an average age of 39 years in the absence of colon surveillance and surgery. Mutations in the APC gene lead to FAP or an attenuated form called AFAP with reduced polyp numbers and cancer risk. Mutation detection fails using DNA based technology in $20 \%$ of FAP and $50 \%$ of AFAP patients due to testing limitations, inability to determine significance of DNA change, or other responsible genes. A subset of disease causing APC mutations may be due to non-coding or even coding variants that result in RNA splice defects.

\section{Methods}

A RNA-based assay has been developed to screen APC mRNA for splice defects in mutation-negative FAP and AFAP patients. Primers and PCR conditions were developed for five overlapping amplicons that cover exons $1-14$ and the beginning of exon 15 in the APC mRNA. PCR products from the cDNA of patients were run on agarose gels and examined for atypical products.

\section{Results}

To date, cDNA from 14 mutation-negative families has been tested, and two mutations resulting in splice defects have been identified when other standard techniques failed to demonstrate loss-of-function mutations. One is a single nucleotide change, deep in intron 4 that generates a splice acceptor site, an additional exon (exon 4A), that includes a new stop codon. The second is a $1.5-\mathrm{kb}$ deletion in intron 14 which causes deletion of exon 14 in the mRNA transcript; exon 13 is spliced to exon 15, resulting in an out-of-frame stop codon. Both families present as AFAP.

\section{Conclusions}

This is a useful assay to compliment DNA-based testing in APC mutation-negative patients or patients with a variant of uncertain significance. Additionally, to address cases where undetected mutations affect the stability of the resultant transcript, allelic imbalance can be examined in amplicons containing one of the common APC single nucleotide polymorphisms (SNPs) using real-time PCR.

\section{Acknowledgement}

This research was supported by NCl grants POI-CA073992 (RWB), ROICA04064l (RWB) and by the Huntsman Cancer Foundation.

\section{Author details}

${ }^{1}$ Huntsman Cancer Institute, University of Utah, Salt Lake City, Utah 84112 , USA. 'Saint Luke's Hospital, Kansas City, Missouri 64111, USA. ${ }^{3}$ Department of Medicine, University of Utah, Salt Lake City, Utah 84112, USA. ${ }^{4}$ Department of Oncological Sciences, University of Utah, Salt Lake City, Utah 84112, USA.

Published: 25 May 2010 